\title{
Asthma and chronic obstructive pulmonary disease guideline implementation: Lessons learned on recruitment of primary care physicians to a knowledge translation study
}

\author{
Louis-Philippe Boulet MD FRCPC ${ }^{1}$, Paul Hernandez MDCM FRCPC ${ }^{2}$, Hollie Devlin $\mathrm{MSC}^{3}$, \\ Marni A Freeman MSc ${ }^{4}$, Samir Gupta MD FRCPC 5
}

L-P Boulet, P Hernandez, H Devlin, MA Freeman, S Gupta. Asthma and chronic obstructive pulmonary disease guideline implementation: Lessons learned on recruitment of primary care physicians to a knowledge translation study. Can Respir J $2013 ; 20(4): 275-280$.

BACKGROUND: Implementation of current clinical practice guidelines in asthma and chronic obstructive pulmonary disease (COPD) is suboptimal. New implementation strategies should be developed and evaluated. METHODS: The authors report the rationale and planned methods of a project that sought to use a multifaceted knowledge translation intervention consisting of interactive education, mentorship through quality circles and practice-based tools in primary care to address key asthma and COPD care gaps. The present study was aborted due to inadequate primary care physician recruitment. Accordingly, the authors provide a critical review of their recruitment strategies and discuss alternative approaches and examples based on previous literature.

DISCUSSION: These practical lessons and discussion seek to inform researchers involved in designing and recruiting for future knowledge translation studies addressing chronic disease management in primary care.

Key Words: Asthma; COPD; Guidelines implementation; Knowledge transfer; Knowledge translation; Medical education; Mentorship; Quality circles

A sthma and chronic obstructive pulmonary disease (COPD) are the two most common chronic pulmonary ailments in Canada, affecting approximately 2.5 million and 750,000 individuals, respectively (1). In the past two decades, the Canadian Thoracic Society has successfully developed and disseminated evidence-based asthma and COPD clinical practice guidelines; however, evidence suggests that guideline implementation for these diseases remains inadequate $(2,3)$.

Successful guideline implementation requires tailoring of selected strategies to settings and population-specific barriers based on established theories and principles $(4,5)$. In 2004, the Canadian Thoracic Society and its collaborators organized a symposium in Quebec City (Quebec) to discuss existing barriers to respiratory guideline implementation and possible knowledge translation (KT) strategies (3). This was followed by an expert-led workshop on guideline implementation strategies in autumn 2007.

Herein, we report the planned methods and outcome of a project that resulted from these meetings. The present study sought to explore the effectiveness of a multifaceted KT strategy in improving concordance with COPD and asthma guidelines among primary care physicians (PCPs) in Canada, but was aborted due to inadequate PCP recruitment. We discuss the difficulties encountered in recruiting $\mathrm{PCPs}$, factors that may have influenced recruitment and alternative strategies. Our goal is to provide practical lessons to inform the design
La mise en ouvre des lignes directrices sur l'asthme et la maladie pulmonaire obstructive chronique : leçons apprises sur le recrutement de médecins de première ligne pour une étude sur le transfert du savoir

HISTORIQUE : La mise en œuvre des lignes de pratique clinique à jour sur l'asthme et la maladie pulmonaire obstructive chronique (MPOC) est sous-optimale. Il faudrait élaborer et évaluer de nouvelles stratégies.

MÉTHODOLOGIE : Les auteurs rendent compte de la raison d'être et de la méthodologie prévue d'un projet qui visaient à faire appel à une intervention de transfert du savoir polyvalente composée d'enseignement interactif, de mentorat par des cercles de qualité et d'outils fondés sur la pratique en première ligne pour corriger les principales lacunes dans les soins de l'asthme et de la MPOC. Cette étude a été annulée en raison du recrutement insuffisant de médecins de première ligne. Par conséquent, les auteurs présentent une analyse critique de leurs stratégies de recrutement et exposent d'autres approches et des exemples fondés sur des publications passées.

EXPOSÉ : Ces leçons pratiques et cet exposé visent à informer les chercheurs qui conçoivent des études de transfert du savoir sur la prise en charge des maladies chroniques en première ligne et qui font du recrutement à cet égard.

of future KT initiatives with similar interventions and/or a similar target audience.

METHODS
Study design
The present study was designed as a two-arm, parallel, randomized,
controlled trial in a primary care setting (Figure 1). Distinct primary
end points were established for asthma and COPD, and sample size
calculations, recruitment estimates and analysis plans were prepared
for each condition.

\section{Interventions}

Standard practice group (control): The investigators planned to mail a copy of the latest Canadian asthma and COPD guidelines to all PCPs in the standard practice (SP) group at the start of the study period. No other intervention would be offered; however, on study completion, all control participants would be offered the educational intervention received by the Targeted Intervention Strategy (TIS) group.

TIS group: The intervention was designed in accordance with best evidence for effective guideline implementation approaches $(4,5)$. PCPs in the TIS group would receive interactive educational interventions; expert mentorship and practice-based tools. The educational interventions consisted of three interactive sessions, two of which would be live meetings of $3 \mathrm{~h}$ duration each, and the third, a $1 \mathrm{~h}$ teleconference

${ }^{1}$ Institut universitaire de cardiologie et de pneumologie de Québec, Université Laval, Québec, Québec; ${ }^{2}$ Department of Medicine, Dalhousie University, Halifax, Nova Scotia; ${ }^{3}$ Integrated Healthcare Communications, Ontario; ${ }^{4}$ GlaxoSmithKline Canada; ${ }^{5}$ The Keenan Research Centre in the Li Ka Shing Knowledge Institute of St Michael's Hospital, University of Toronto Department of Medicine, Toronto, Ontario Correspondence: Dr Louis-Philippe Boulet, Institut universitaire de cardiologie et de pneumologie de Québec, 2725, Chemin Sainte-Foy, Québec,

Québec G1V 4G5. Telephone 418-656-4747, fax 418-656-4762, e-mail lpboulet@med.ulaval.ca 


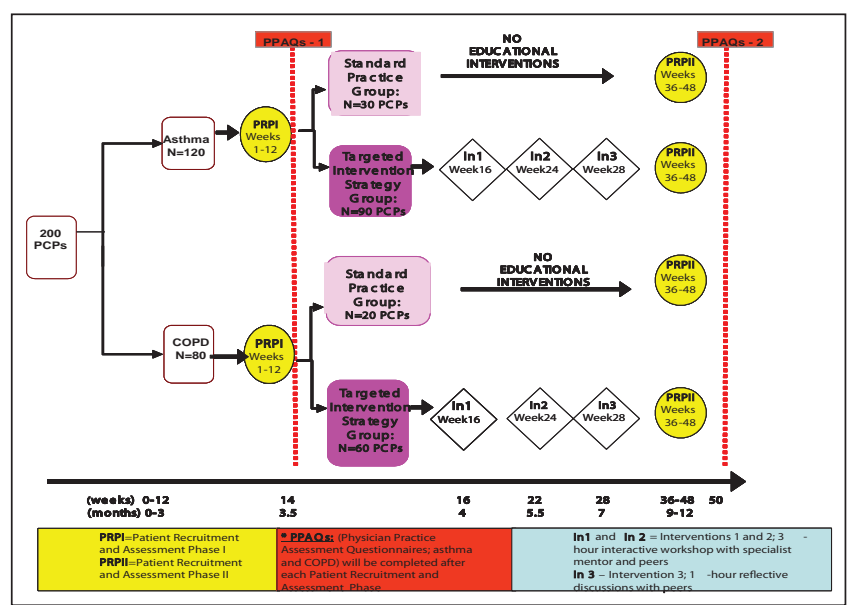

Figure 1) Study design algorithm. COPD Chronic obstructive pulmonary disease; PCP Primary care physician

(occurring at weeks 16, 24 and 28, respectively) (Figure 1). The content of the educational interventions is detailed in Table 1. For expert mentorship, each PCP would be assigned to a specialist mentor and each mentor would lead a group of four to six PCPs (ie, a 'quality circle'). Members of each quality circle would also participate in the educational interventions together. Groups would self-define if and how they wished to communicate with one another and with their mentor in between sessions (ie, through e-mail, telephone, etc). Finally, a number of practice-based tools would be provided at the first educational intervention session. These included copies of the most recent Canadian asthma and COPD guidelines, a list of useful websites and local resources for health care professionals and patients (eg, referral forms for local pulmonary rehabilitation programs, smoking cessation programs and pulmonary function laboratories), copies of existing action plans for asthma and COPD, office reminder tools and existing practice-based algorithms. The summary of the above information was provided to the recruited participants to explain the nature of the study and the requirements for their participation.

\section{Study population}

Mentors (respirologists): A convenience sample of potential mentors was identified by the principal investigators (PIs). They consisted of practicing respirologists with an interest in continuing medical education (CME) who were not involved in respiratory guidelines development.

Mentees (PCPs): PCPs were eligible if they managed at least 15 patients with asthma and 15 patients with COPD per month in an office setting; and agreed to designate an appropriately trained person at his/her site to identify eligible study patients, collect consent and provide a questionnaire to patients. Physicians were excluded if they had presented at a CME event on asthma or COPD in the past year; had completed any specialty training in respiratory diseases; or practiced primarily at walk-in clinics, with children or in emergency departments.

Patients: Patients were eligible if they spoke French or English; and had a diagnosis of asthma or COPD (as per their participating PCP). Patients with asthma had to be between 18 and 45 years of age and patients with COPD had to be between 40 and 75 years of age and have a smoking history of $>10$ pack-years. Patients were excluded if they had any condition that could interfere with study measurements (as per their participating PCP); had any known respiratory disorders other than asthma or COPD; or were currently participating in another clinical trial.

\section{Recruitment strategy}

All physician recruitment was conducted with the help of an independent contract research organization (CRO).
TABLE 1

\section{Educational sessions}

Session 1 (in-person meeting at 16 weeks, $3 \mathrm{~h}$ duration)

Welcome, round table introductions, agenda and objectives of the meeting

General presentation

Spirometry interpretation

Why/when/how to perform spirometry

Inhaler technique instruction

\section{Asthma presentation}

Key recommendations and messages in Canadian asthma guidelines

Written asthma action plans (why/when/how to make one)

Therapeutic algorithm (including recommendation for maintenance antiinflammatory medication if a patient has symptoms or is using a rescue inhaler more than twice/week [except for use 1/day befiore exercise]) Guideline-recommended criteria for assessment of control

\section{COPD presentation}

Key recommendations and messages in Canadian COPD guidelines

Exacerbation assessment/what is an exacerbation

Assessment of level of function and disability (Modifed MRC scale)

Therapeutic algorithm according to MRC class (including a recommendation for long-acting bronchodilators for patients in MRC class $>3$ )

Smoking cessation counselling techniques, pharmacotherapy review, review of evidence supporting a $30 \mathrm{~s}$ smoking cessation intervention

\section{Workshop 1: Spirometry}

Sample spirometry results provided for analysis and discussion (assessment of adequacy of test, interpretation for diagnosis and for routine follow-up, how results should impact treatment)

Each group to compile list of locations where spirometry is available in their region (to be provided to physicians not performing office spirometry)

\section{Workshop 2: Inhaler technique}

Show-and-tell with various inhalers

Share best practices on testing patients' inhaler technique

Group to compile a list of certified asthma educators available in region (to be provided to physicians who do not have current regular access to certified asthma educators)

Break

\section{Workshop 3: Practice tools}

Introduction

How/when to use them

Workshop 4: Barriers to implementation

Identify current barriers to implementation of each of the key guideline recommendations

Share current best practice tips to overcome barriers

Wrap-up and next steps in study

Session 2 (in-person meeting at 24 weeks, 3 h duration)

Welcome, round table introductions, agenda, objectives of the meeting

Summary of key recommendations and messages (asthma + COPD)

Open discussion about implementation barriers and facilitators for key guideline recommendations (as driven by participants)

\section{Break}

Report by physicians on their experience with implementation of key guidelines recommendations in their practice

Open discussion on the use of tools and barriers and facilitators to their use General discussion and conclusions

Session 3 (teleconference at 28 weeks, $1 \mathrm{~h}$ duration)

Welcome, round table introductions, agenda and objectives of the teleconference

Questions for discussion would be provided in advance of teleconferences

Facilitated discussion on impact on practice from what was learned during the study

Summary and conclusions

COPD Chronic obstructive pulmonary disease; MRC Medical Research Council 
TABLE 2

Primary care physician (PCP) recruitment stages

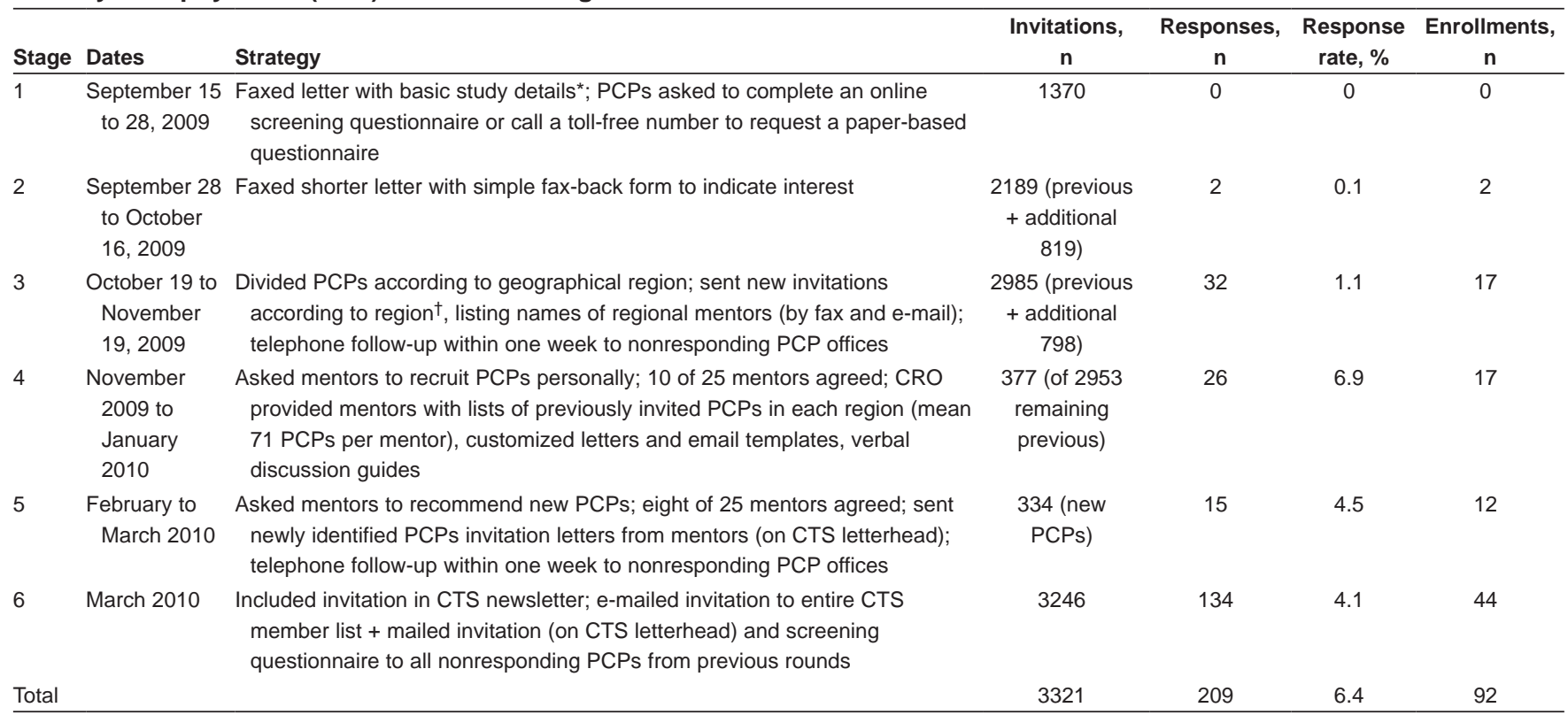

*Included the purpose of the study, estimated time requirements ( $7 \mathrm{~h}$ in educational sessions and $2.5 \mathrm{~h}$ to $3 \mathrm{~h}$ for patient for recruitment, consent, and all questionnaires [approximately 10 min per patient $\times 15$ patients] [which could be accomplished by any office staff]), the number of patients to be recruited, remuneration ( $\$ 40$ per patient recruited $\times 30$ patients; therefore, up to $\$ 1,200$ over the one-year period) and potential for continuing medical education credits (up to 7 Mainpro-M1 credits through the College of Family Physicians of Canada for time spent in the mentorship groups)]; ${ }^{\dagger}$ Regions were as follows: western Canada, Ontario, Quebec and Atlantic Canada. CRO Contract research organization; CTS Canadian Thoracic Society

Respirologist recruitment (mentors): Candidates were e-mailed a letter from the PIs explaining the study goals along with mentor roles and remuneration ( $\$ 1,500$ for each of sessions 1 and $2, \$ 300$ for session 3 ) (Table 1), estimated time requirements (seven total hours in the educational sessions plus any additional mentoring time), and contact information for more information. Candidates were asked to accept or decline study participation via a reply e-mail to the CRO. Nonresponders were prompted by the CRO two to three weeks after the initial invitation, and then recontacted directly by the study's PIs if they did not respond within five to six weeks.

PCP recruitment (mentees) (Table 2): Recruitment occurred over a seven-month period (September 2009 to March 2010). PCPs were recruited from across Canada, from a list purchased from a private company (Professional Targeted Marketing, Markham, Ontario). Study locations were established based on mentor practice addresses, and postal code matching randomly identified potential mentees practicing within a $1 \mathrm{~h}$ drive from each location.

\section{Outcomes}

Primary outcomes: The coprimary outcomes (for asthma and COPD) were defined as the change in patient-reported physician conformity to each of the following key guideline recommendations, measured through a questionnaire:

A. For asthma, assessment of asthma control, defined as ascertainment of at least two of the five asthma control questions recommended by the Canadian Asthma Guidelines (6), including daytime asthma symptoms, night-time asthma symptoms, use of asthma rescue medication, limitations in daily activities and frequency of asthma attacks.

B. For COPD, assessment of breathlessness threshold, defined as ascertainment of at least two of the following four states: at rest, walking, with exercise and with any other type of activity.

The difference in the change in the percentage of patients appropriately assessed between baseline (patient recruitment phase-I [PRP-I]) and end-of-study (PRP-II) (12 months) would be compared between the TIS and SP groups (Figure 1).
Secondary outcomes: Secondary outcomes collected through the patient questionnaires included other guideline-recommended physician behaviours. For asthma, these included spirometry performance, asthma action plan provision and review, inhaler technique review, and maintenance anti-inflammatory medication prescription when indicated. For COPD, these included spirometry performance, inhaler technique review, exacerbation screening, long-acting bronchodilator prescription when indicated and smoking cessation efforts in smokers. A separate questionnaire measured physician self-reported changes in knowledge and adherence to guideline-recommended behaviours (the Physician Practice Assessment Questionnaire [PPAQ]) (7).

\section{Study procedures}

Each PCP would be assigned to either the asthma or COPD arm of the study and asked to recruit 15 consecutive clinic patients, regardless of the reason for the clinical visit. Patients would not be followed over time, but 15 new patients would be recruited at each of the two recruitment phases. Stratified physician randomization would randomly assign $75 \%$ of PCPs to the TIS group and $25 \%$ to the SP group.

Data collection requirements

PCPs were asked to delegate a clinic employee to identify, acquire consent from and provide questionnaires to eligible patients. Immediately following the physician visit, patients would complete and return the questionnaire in a sealed postage-paid envelope that the physician's designated employee would then mail to the CRO. PCPs would also complete and mail in the PPAQ at the conclusion of each recruitment phase (PRP-I and PRP-II). These procedures would be identical for physicians in the control and intervention groups.

\section{Sample size calculations}

Sample size calculations were based on a two-sided score test for cluster randomization (8) with $90 \%$ power, an intracluster correlation of 0.1 and an overall significance level of 0.05 .

For asthma, it was estimated that $28 \%$ of patients currently have their asthma control assessed by PCPs in accordance with asthma 
guidelines $(9,10)$. Assuming a $5 \%$ improvement from baseline in the SP group and a $15 \%$ improvement in the TIS group, $120 \mathrm{PCPs}$ and 3600 patients would be required to detect this difference in improvement between groups with the desired allocation ratio of $1: 3$ between the SP and TIS groups (ie, 30 PCPs randomly assigned to the SP group and 90 PCPs to the TIS group).

For COPD, it was estimated that only $7 \%$ of Canadian physicians currently assess breathlessness with activity (11). Assuming a 5\% improvement from baseline in the SP group and an $18 \%$ improvement in the TIS group, 80 PCPs and 2400 patients would be required to detect this difference in improvement between groups with the $1: 3$ allocation ratio (ie, 20 PCPs randomly asigned to the SP group and $60 \mathrm{PCPs}$ to the TIS group).

\section{Feasibility}

In accordance with sample size calculations, the objective was to include 200 PCPs, recruiting 15 subjects each at PRP-I and PRP-II, for a total of 6000 patients. Desiring quality circle sizes of four to five mentees per mentor, the objective was to recruit 40 to 50 specialist mentors.

It was believed that patient recruitment was feasible based on both prevalence data and previous recruitment to similar programs. The prevalence of asthma in Canadian adults is approximately $8.4 \%$ (12). Assuming an average practice size of 1200 patients and once per year asthma follow-up (a conservative estimate), 101 unique patient asthma visits per year and 23 visits per 12-week recruitment phase was estimated. In a previous Canadian primary care asthma study requiring both physician and patient questionnaire components (requirements similar to the present study) (10), each PCP successfully recruited and assessed a median of 26 patients with asthma over a 12 -week period.

Similarly, the prevalence of COPD in Canadian adults is approximately $8.0 \%$ (13). As above, assuming a practice size of 1200 patients and one visit per year, it was estimated that each physician would see 96 patients each year and 22 patients during each 12 -week recruitment phase.

\section{Specialist recruitment}

\section{RESULTS}

Between September 2009 and January 2010, 25 of 30 (83\%) contacted respirologists were successfully recruited. A second mentor recruitment phase was delayed when PCP recruitment was noted to be slow and was eventually aborted.

\section{PCP recruitment (Table 2)}

Six recruitment stages were completed between September 2009 and March 2010. A total of 92 of 209 (44\%) responding PCPs were eligible and recruited. Of these, $28 \%$ were female and $54 \%$ practiced in large urban centres.

At that stage, it was decided to abort the study due to inadequate recruitment. Although qualitative data to assess reasons for failed recruitment were not collected, reasons for nonparticipation communicated actively by PCPs included: lack of time; perception that the study was too complex; insufficient financial incentive; and concurrent participation in other studies.

\section{DISCUSSION}

We have presented a detailed protocol designed to evaluate a multifaceted KT initiative targeting gaps in primary care asthma and COPD management. Our intervention consisted of a combination of interactive education, mentorship in quality circles and practice-based tools. Unfortunately, the study was aborted due to insufficient PCP recruitment.

Recruitment of PCPs to research studies is a well-recognized obstacle faced by KT researchers; however, limited empirical data regarding the effectiveness of various recruitment strategies are available to guide research design (14). Accordingly, it is worthwhile considering which factors may have affected recruitment to our study in the context of available evidence.
Our recruitment approach began with a mass fax 'blast' in the first round, followed by an additional fax in the second round. We attempted to minimize the time, cost and effort required for PCPs to respond by offering an online eligibility questionnaire, a toll-free number and a fax-back form. In a retrospective analysis of strategies used to recruit PCPs to a trial of cardiovascular disease management tools, Ellis et al (15) noted that such strategies, in which potential participants were required to actively opt-in to the study, were universally less effective than strategies in which participants had to actively optout. Similar to our study, recruitment rate for mass fax was $0.33 \%$, e-mail was $0.94 \%$ and opinion leader e-mail was $1.02 \%$. Conversely, recruitment rates for opt-out strategies were as follows: cold calls (5.35\%), in-person practice presentations $(41.7 \%)$ and strategies in which a personal (in-person or telephone) invitation was made by someone who had a pre-existing relationship with the potential recruit (33.3\%) (15).

In particular, the 'physicians-recruiting-physicians' method, in which local physician leaders are used to recruit practices, has been noted to produce very high response rates (15). Although these strategies are more labour intensive than most opt-in strategies, their higher success rates resulted in a lower total cost per participant recruited (15). Although we did call physicians' offices after the third and fifth rounds, these were follow-up calls to ascertain whether they had received study information, as opposed to 'cold calls' for study solicitation. In the fifth round, we sought to leverage existing relationships by asking mentors to target PCPs whom they knew, but again used an opt-in (mailed letter) as opposed to an opt-out (personal visit or telephone call) strategy. Conversely, we did use an opt-out strategy for mentor recruitment, whereby nonresponders were called directly by the study's PIs. This may, in part, explain why mentor recruitment rates were dramatically higher.

Next, the controlled study design may have discouraged participation because control participants had the same reporting burden as active participants without the benefit of the intervention. To mitigate this, we minimized the number of PCPs to be enrolled in the control group (using a 3:1 allocation ratio), and offered all control participants access to the intervention after study completion. Unfortunately, these design aspects were not communicated in initial invitation letters and these issues may have contributed to PCP reluctance. An alternative approach that may have been considered is the stepped-wedge trial design, in which the intervention is rolled-out sequentially over a number of time periods, such that all participants had received the intervention by the end of the study. This design may not only improve recruitment by guaranteeing all participants the benefit of the intervention, but also enables modelling of the effect of time on the effectiveness of the intervention. Disadvantages include increased study time requirements, possible concurrent increases in cost, and lack of familiarity with this design among the study's target audience members.

Next, we must consider the intervention itself. The content of the intervention was unlikely to be a major limiting factor because previous needs assessments have shown that Canadian PCPs are particularly interested in enhancing their knowledge and management of asthma and COPD. Although previous large-scale studies have successfully recruited physicians to quality circle-based interventions, qualitative research has suggested that participants in quality improvement initiatives have a strong preference for involvement in the design of the intervention itself, rather than being asked to abide by a predesigned strategy (16). Our interventions and tools were designed and delivered centrally using a 'top-down' approach based on research evidence and expert opinion (16). A 'local' participatory approach that enables frontline staff members to mould the intervention to their specific context and needs may have facilitated buy-in and has been shown to be intrinsically more rewarding for participants (16).

Furthermore, our protocol called for patients to independently report on physician behaviour through a patient questionnaire, in addition to a self-reported physician behaviour questionnaire. Some 
physicians may have perceived this to be a test of their behaviour and a sign of distrust on behalf of the researchers. Physicians may also have had concerns that this audit could compromise their patients' trust in the care that they provided. Conversely, qualitative studies have indicated that physicians perceive great benefit from audit practices as long as private feedback is included, and can use these data effectively to drive autonomous practice change (17). Accordingly, it is possible that inclusion of a feedback mechanism in our study design would have improved PCP perception of the study, augmenting recruitment.

Study remuneration is another consideration. We offered physicians $\$ 40$ per recruited patient. At an estimated $10 \mathrm{~min}$ per patient (including recruitment, consenting, questionnaire provision and completion of the PPAQ by the physician), this equates to $\$ 240 / \mathrm{h}$, which is similar to the average hourly uninsured medical services rate of $\$ 243.98$ recommended by the Ontario Medical Association. Given that most of these tasks could have been completed by nonphysician staff, we believe that this was a generous reimbursement. A higher remuneration threshold would have amounted to an inducement. Evidence for the effect of inducements on recruitment rates is lacking, and they risk creating a selection bias for the minority of PCPs (estimated at 15\%) who are motivated to participate in research by financial incentives (18). In fact, qualitative data suggest that while proper reimbursement for time spent is important, financial inducements above and beyond this are not considered to be important by most PCPs (18). Nonfinancial factors that are likely more important include their interest in the research topic and aims of the study, interest in improving clinical practice and introducing new ideas, professional obligation, the possibility of medical care benefits associated with participation, and personal acquaintance with the researcher $(15,18)$. Also, we did not specifically remunerate physician time spent in educational aspects of the intervention, but did offer CME credits for this time. Unfortunately, educational credits have been shown to be an important motivator for study participation in only $20 \%$ of PCPs (15).

The data collection burden imposed by the study may also have been a factor. Patient recruitment and questionnaires were to be completed during normal clinic hours by the PCP or a designated staff member, threatening to impede workflow and efficiency. In a study examining predictors of PCP participation in randomized controlled trials, the most common reason for nonparticipation was difficulty in coping with the organizational requirements of the study (19). These 'internal practice issues' were more commonly cited (38\% of practices) than factors relating to the study itself (7\%) (19). This may have been another factor in our high mentor recruitment rates, given that mentors were offered a similar hourly reimbursement rate but had no practice-related burdens. In recruitment to trials involving patients with Alzheimer's disease, most PCPs initially believed that their staff burdens would increase prohibitively, but changed their minds after a brief educational session (14). Strategies to improve recruitment to our trial could have included specifically targeting clinics with existing nursing support, designating a portion of the remuneration to compensate for increased administrative staff hours, and specifically educating physicians about actual increases in personal staff workload required by the study.

It is worth comparing with a previous Canadian study that successfully recruited a large number of PCPs to a similar intervention. Ioannidis et al (20) recruited 340 PCPs from across Canada to a twoyear quality circle intervention in osteoporosis care (20). Their multifaceted KT intervention was comparable with our own, with serial group physician education workshops led by local primary care physicians or a regional specialist (quality circles), practice-based printed educational materials and practice audits by physicians (20). Physicians were awarded CME credits based on time spent with mentors, remuneration was considered to be 'nominal' and clinic staff identified prospective patients. However, PCPs were recruited primarily based on a list of colleagues known to facilitators of each quality circle (a 'physicians-recruiting-physicians' strategy), and follow-up contact involved an opt-out rather than an opt-in strategy (facilitators or the
TABLE 3

Factors affecting primary care practitioner participation in practice-based implementation studies

Limiting factors
Ineffective recruitment strategies
'Opt-in' recruitment strategies (mail, fax, e-mail, online recruitment)
'Passive' methods of recruitment (announcements, etc)
Design related
Inclusion of a control arm in the study design
Inclusion of practice audits (may be perceived as external criticism
of practice)
Inclusion of patient report of physician behaviour (may be perceive
undermine patient-clinician trust)
Burden of data collection (may impede workflow and efficiency)
Administrative burden on clinical staff
Others
Lack of interest about the disease or domain examined
Competition with other research studies

Facilitating factors

Effective recruitment strategies

'Opt-out' strategies (in-person or telephone recruitment)

'Physicians-recruiting-physicians'

Leveraging a personal acquaintance between the researcher and the participant

Design related

Use of skewed allocation to minimize clinicians enrolled to the control group

Use of special designs to ensure access to the intervention for all clinicians (eg, stepped-wedge trial design)

Engagement of participants from study inception, including in intervention design

Inclusion of personal and private feedback when employing practice audits

Enabling physicians to anonymously compare their performance to that of their peers when employing practice audits

Reimbursement related

Reimbursement for time spent commensurate with lost wages (financial inducements above and beyond this is ineffective in most primary care physicians)

Dedicated reimbursement for all administrative activities

Participant related

Targeting participants in clinics with existing administrative support

Targeting participants with an intrinsic interest in the research topic and aims of the study

Leveraging the intervention's ability to improve clinical practice and medical care for participants

Specifically educating physicians about the nature and extent of the study's administrative burden

Other

Use of a pilot study to optimize recruitment, study materials and the intervention

project manager directly telephoned prospective recruits) (20). The present study also made use of physician audit data both in educational sessions and for direct physician feedback, enabling physicians to anonymously compare their practices with those of their peers. Authors believed that the audit and feedback component was a major contributor to physician participation and satisfaction with the intervention. These authors also initially studied their intervention in a pilot sample of 52 PCPs, enabling them to test the effectiveness and feasibility of recruitment. Qualitative data from pilot participants guided improvements to project design, including using facilitators more effectively in the recruitment process by building on their local networking capabilities, and changes to meeting format and content and facilitator roles to improve participant engagement. 


\section{SUMMARY}

We designed a complex KT intervention study for primary care that was abandoned due to inadequate PCP recruitment. We reviewed which factors likely limited recruitment to the present study and which factors may facilitate recruitment, according to best evidence (Table 3). Future KT studies using similar interventions and with a similar target audience should consider using opt-out type recruitment approaches, carefully targeting practices with appropriate administrative support, specifically remunerating study-related administrative work and actively educating PCPs about the nature of the administrative burden of study participation. A participatory approach to intervention development and efforts to include an audit and anonymous feedback mechanism in the study design may also improve participant

\section{REFERENCES}

1. Canadian Institute for Health Information, Canadian Lung Association, Health Canada, Statistics Canada. Respiratory Disease in Canada, 1st edn. Ottawa: Health Canada, 2001:1-1.

2. Chapman KR, Ernst P, Grenville A, Dewland P, Zimmerman S. Control of asthma in Canada: Failure to achieve guideline targets. Can Respir J 2001;8(Suppl A):35A-40A.

3. Boulet LP, Becker A, Bowie D, et al. Implementing practice guidelines: A workshop on guidelines dissemination and implementation with a focus on asthma and COPD. Can Respir J 2006;13(Suppl A):A5-A47.

4. Strauss SE, Tetroe JM, Graham ID. Knowledge translation is the use of knowledge in health care decision making. J Clin Epidemiol 2011;64:6-10.

5. Davies P, Walker AE, Grimshaw JM. A systematic review of the use of theory in the design of guideline dissemination and implementation strategies and interpretation of the results of rigourous evaluations. Implement Sci 2010;5:14.

6. Lougheed MD, Lemière C, Dell SD, et al. Canadian Thoracic Society Asthma Management Continuum - 2010 consensus summary for children six years of age and over, and adults. Can Respir J 2010;17:15-24.

7. Boulet LP, Devlin H, O'Donnell DE. The Physicians' Practice Assessment Questionnaire on asthma and COPD. Respir Med 2011;105:8-14.

8. Donner A, Klar N. Design and Analysis of Cluster Randomization Trials in Health Research. London: Hodder Arnold Publication, 2000:192.

9. Fitzgerald JM, Boulet LP, McIvor RA, Zimmerman S, Chapman KR. Asthma control in Canada remains suboptimal: The Reality of Asthma Control (TRAC) study. Can Respir J 2006;13:253-9.

10. Chapman KR, Boulet LP, Rea RM, Franssen E. Suboptimal asthma control: Prevalence, detection and consequences in general practice. Eur Respir J 2008;31:320-5. buy-in. Novel designs, such as the stepped-wedge trial design, may be beneficial when a control arm is included, and researchers may consider a pilot trial to examine recruitment feasibility before embarking on a large-scale study.

ACKNOWLEDGEMENTS: The authors sincerely thank Denis O'Donnell, Shawn Aaron, Kenneth Chapman, Diane Lougheed, Darcy Marciniuk and Michel Rouleau for their input into this project. All authors have contributed to the protocol and the writing of this article.

FUNDING: This research program conducted with the Canadian Thoracic Society was supported by GlaxoSmithKline Canada.

11. Chronic Obstructive Pulmonary Disease (COPD). A National Report Card. Canada. 2005. <www.lung.ca/_resources/2005.copd_ reportcard.pdf> (Accessed August 21, 2008).

12. Public Health Agency of Canada. Life and Breath: Respiratory Diseases in Canada, 2007. Ottawa: Public Health Agency of Canada; 2007.

13. Canadian Lungs Association. Chronic Obstructive Pulmonary Disease (COPD): A National Report Card, 2005. Canadian Lung Association, 2005.

14. Carr SA, Davis R, Spencer D, et al. Comparison of recruitment efforts targeted at primary care physicians versus the community at large for participation in Alzheimer disease clinical trials. Alzheimer Dis Assoc Disord 2010;24:165-70.

15. Ellis SD, Bertoni AG, Bonds DE, et al. Value of recruitment strategies used in a primary care practice-based trial. Contemp Clin Trials 2007;28:258-67.

16. Parker LE, de Pillis E, Altschuler A, Rubenstein LV, Meredith LS. Balancing participation and expertise: A comparison of locally and centrally managed health care quality improvement within primary care practices. Qual Health Res 2007;17:1268-79.

17. Spooner A, Chapple A, Roland M. What makes British general practitioners take part in a quality improvement scheme? J Health Serv Res Policy 2001;6:145-50.

18. Draper H, Wilson S, Flanagan S, Ives J. Offering payments, reimbursement and incentives to patients and family doctors to encourage participation in research. Fam Pract 2009;26:231-8.

19. Down L, Metcalfe C, Avery K, et al. Factors distinguishing general practitioners who more readily participated in a large randomized trial were identified. J Clin Epidemiol 2009;62:67-73.

20. Ioannidis G, Papaioannou A, Thabane L, et al. The utilization of appropriate osteoporosis medications improves following a multifaceted educational intervention: The Canadian quality circle project (CQC). BMC Med Educ 2009;9:54. 


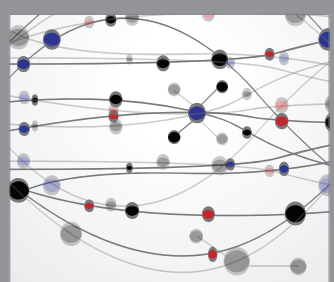

The Scientific World Journal
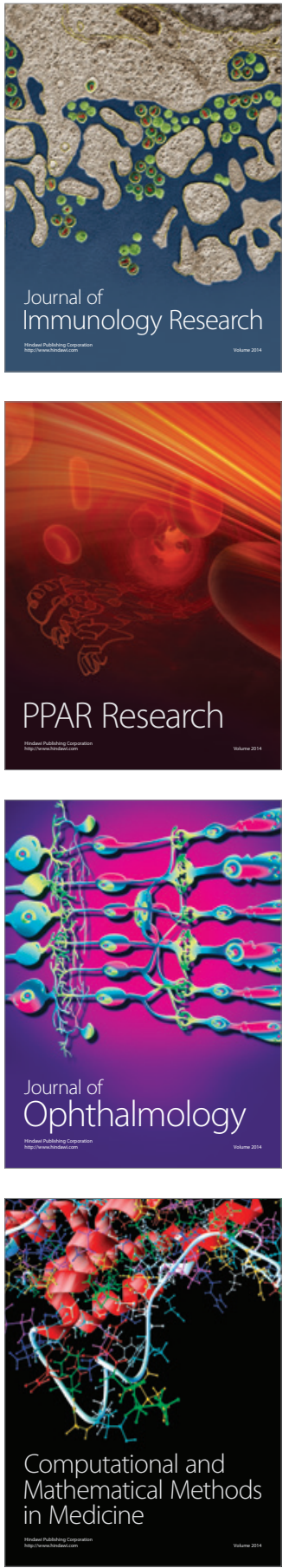

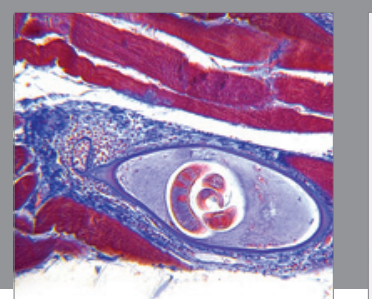

Gastroenterology Research and Practice

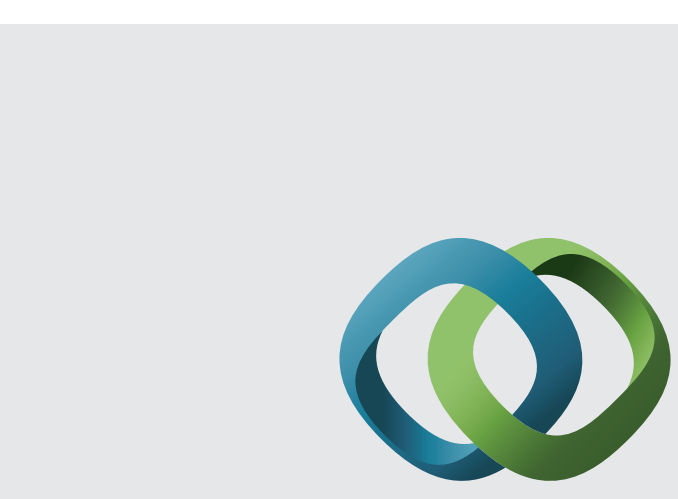

\section{Hindawi}

Submit your manuscripts at

http://www.hindawi.com
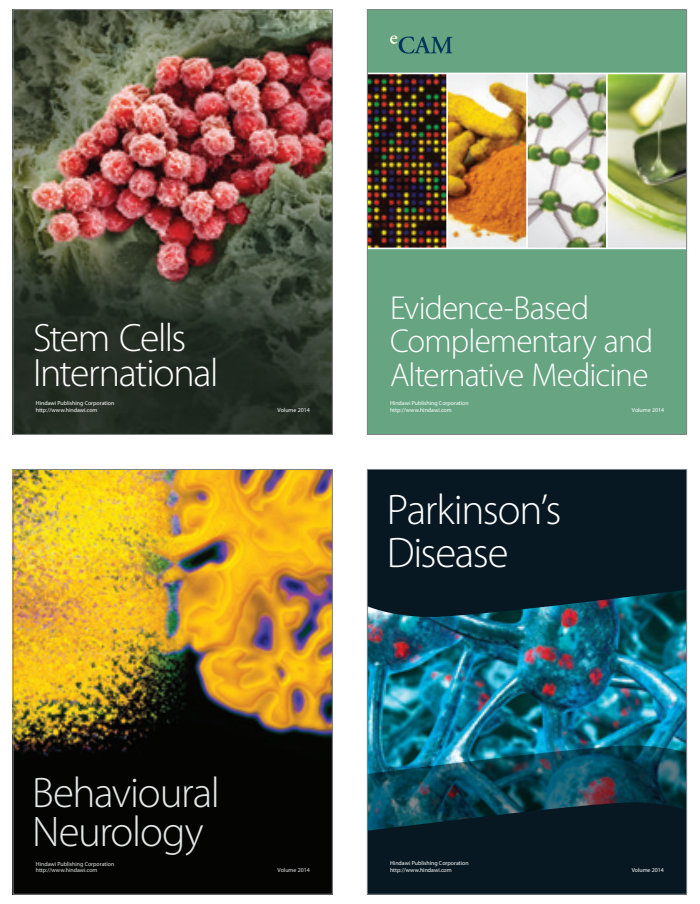
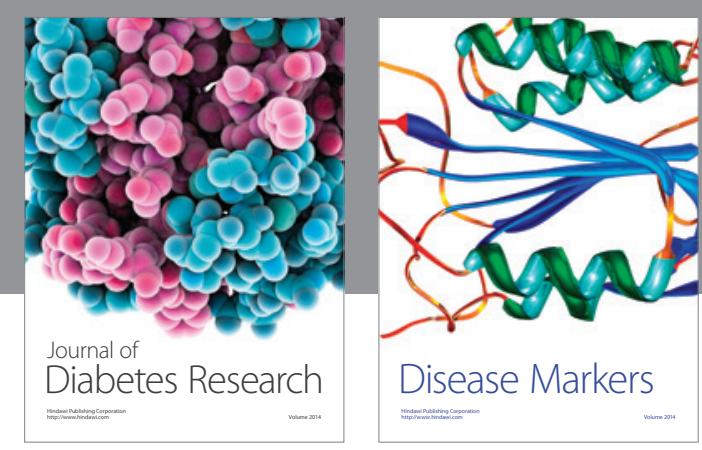

Disease Markers
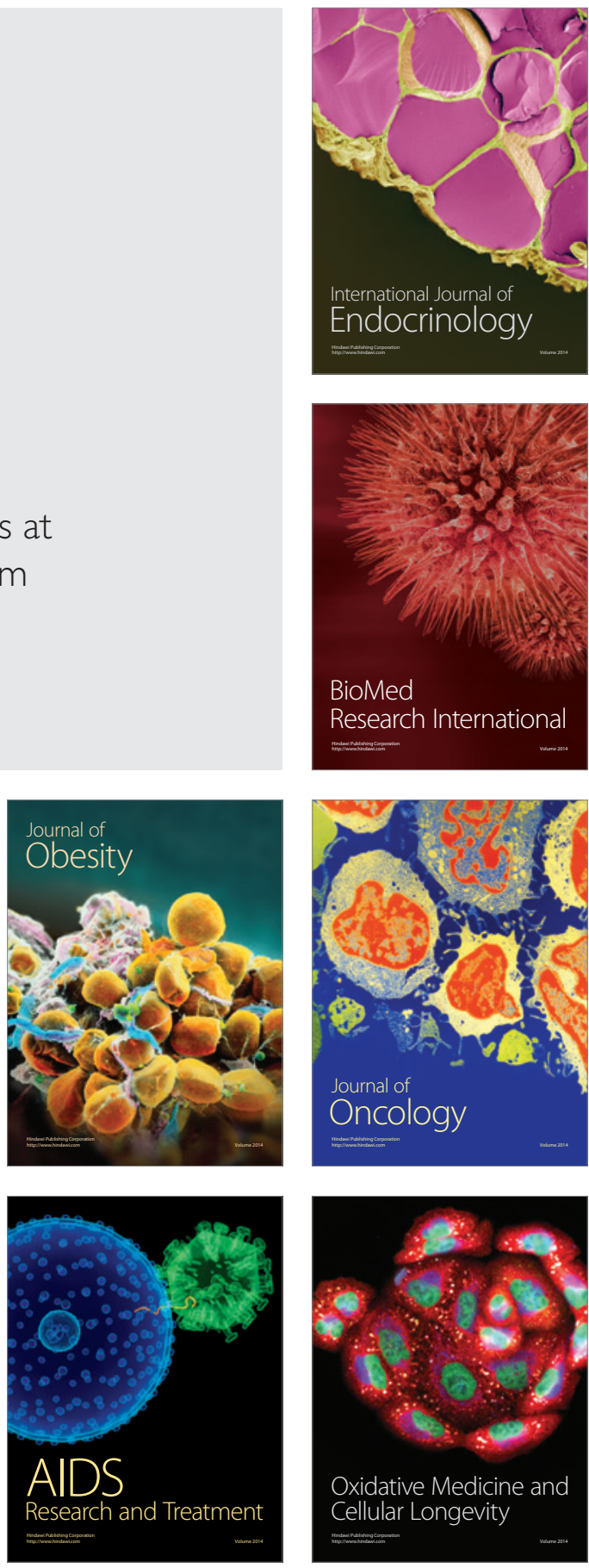